\title{
On Causality and the Impulse Response Scandal
}

\author{
IRWIN W. SANDBERG \\ Department of Electrical and Computer Engineering \\ The University of Texas at Austin \\ 1 University Station C0803 \\ Austin, Texas $78712-0240$ \\ USA
}

\begin{abstract}
In a recent study of a family of continuous input-output maps representing linear shift-invariant systems that take a set of bounded uniformly-continuous signals into itself, it is shown that the family contains causal maps whose impulse response is the zero function, but which take certain inputs into nonzero outputs. Here we show that a similar result holds for maps whose domain is the whole space of bounded Lebesguemeasurable signals, and whose range is contained in a set of bounded uniformly-continuous functions.
\end{abstract}

Key-Words: - linear systems, impulse responses, causality, bounded inputs.

\section{Introduction}

In the signal-processing literature, $x(t)$ typically denotes a function. In the following we distinguish between a function $x$ and $x(t)$, the latter meaning the value of $x$ at time $t$. Sometimes a function $x$ is denoted by $x(\cdot)$, and also we use $H x$ to mean $H(x)$. This notation is often useful in studies of systems in which signals are transformed into other signals.

Consider a continuous-time linear shift-invariant system governed by the relation $y=H x$ in which $x$ is an input, $y$ is the corresponding output, and $H$ is the system map that takes inputs into outputs. Assume that inputs and outputs are complex-valued functions defined on the set $\mathbb{R}$ of real numbers. As is well known, it is part of the engineering folklore that the input-output properties of $H$ are completely described by its impulse response. Using a standard interpretation of what is meant by a system's impulse response, it is shown in [1] that this part of the engineering folklore is incorrect in the simple setting in which $x$ is drawn from the linear space $\mathcal{C}$ of bounded uniformlycontinuous complex-valued functions defined on $\mathbb{R}$, and

(i) $H$ is a map from $\mathcal{C}$ into $\mathcal{C}$. That is, the system takes bounded uniformly-continuous inputs ${ }^{1}$ into bounded uniformly-continuous outputs.

(ii) $y$ depends continuously on $x$ with respect to the usual sup norm. In other words, $H$ is continuous with respect to the norm given by

$$
\|w\|=\sup _{t \in \mathbb{R}}|w(t)| .
$$

\footnotetext{
${ }^{1}$ A complex-valued function $x$ defined on $\mathbb{R}$ is uniformly continuous if for each $\epsilon>0$ there is a $\delta>0$ for which $\left|x\left(t_{1}\right)-x\left(t_{2}\right)\right|<\epsilon$ whenever $\left|t_{1}-t_{2}\right|<\delta$. In applications, inputs are often uniformly continuous. For example, continuous almost periodic functions [2] are uniformly continuous. So are bounded continuous functions $x$ for which both $\lim _{t \rightarrow \infty} x(t)$ and $\lim _{t \rightarrow-\infty} x(t)$ exist.
}

(iii) $H$ is causal in the usual sense.

More specifically, it was shown that there is an $H$ of the kind described above whose impulse response is the zero function, but which takes certain inputs into nonzero outputs. ${ }^{2}$ Here, in the following section, we show that a similar result holds for continuous inputoutput maps whose domain is the whole space of bounded Lebesgue-measurable functions, and whose range is contained in $\mathcal{C}$. This result provides significant additional insight, because system inputs that need not be uniformly continuous are often of interest, and the maps to which we direct attention are reasonable - in the sense that both the input and output spaces are spaces of bounded measurable functions. ${ }^{3}$ (As is well known, bounded measurable functions have the important property that they are integrable over any finite subinterval of $\mathbb{R}$.) Also given in the next section are some related comments concerning the frequency response of linear systems.

\section{Input-Output Maps and Impulse Responses}

We begin with the comment made in [1] that, as is well known, the concept of an impulse function as described by P. Dirac, while often useful in engineering and scientific applications, is unsatisfactory from the viewpoint of mathematics. It is unsatisfactory

\footnotetext{
${ }^{2}$ For related results, concerning discrete-time systems, including a representation theorem for input-output maps, see [3] and Appendix G of [4]. For material related in a general sense concerning discrete-time systems, see [5] and [6]. See also [7], which addresses continuous-time and continuous-space cases. It appears that as early as 1932 Banach was aware of the lack of existence of generalized convolution-sum representations for certain linear system maps (see [8, pp. 158, 159]). For further material concerning discrete-time systems, in the context of the theory of conjugate spaces, see e.g., [9, p. 228, Table 1 and p. 229, Exercise 9].

${ }^{3}$ Some observations concerning extensions are made in [1], but the important case addressed here is not considered.
} 
because according to the usual theory of integration,

$$
\int_{\mathbb{R}} q(t) d t=0
$$

for any function $q$ with $q(t)=0$ for $|t|>0$, even if $q(0)=\infty$ is allowed. ${ }^{4}$ It is also well known that one alternative approach (see, for example, [10]) involves envisioning a sequence of progressively taller and narrower unit-area functions centered at $t=0$. Thus, with $\Lambda$ the set $(0,1)$ or the set $\left\{n^{-1}, n=1,2, \ldots\right\}$, let $\left\{q_{\lambda}: \lambda \in \Lambda\right\}$ be a family of bounded complex-valued Lebesgue-integrable functions defined on $\mathbb{R}$ such that

$$
\int_{\mathbb{R}} q_{\lambda}(t) d t=1
$$

for all $\lambda$. Assume that, in some precise sense, the $q_{\lambda}$ are progressively taller and narrower as $\lambda \rightarrow 0$. For example, we can take $\Lambda=(0,1)$ or $\left\{n^{-1}, n=1,2, \ldots\right\}$ with $q_{\lambda}$ given by the familiar expression

$$
\begin{aligned}
q_{\lambda}(t) & =1 / \lambda, \quad|t| \leq \lambda / 2 \\
& =0, \quad \text { otherwise. }
\end{aligned}
$$

We say that $H$ has an impulse response if $\lim _{\lambda \rightarrow 0}\left(H q_{\lambda}\right)(t)$ exists as a complex number for each $t$, and if $H$ has an impulse response, this limit is taken to be the impulse response.

In this section we view $\mathcal{C}$ as a normed linear space over the complex numbers, with the norm $\|\cdot\|$ given by (1). Let $L_{\infty}(\mathbb{R})$ stand for the normed linear space of bounded Lebesgue measurable complex-valued functions defined on $\mathbb{R}$, with the norm also given by (1), and let $\mathcal{H}$ denote the family of all continuous linear shift-invariant maps $H$ from $L_{\infty}(\mathbb{R})$ into $\mathcal{C}$. By $H \in \mathcal{H}$ is causal is meant that $H$ is causal in the usual sense that for each $t$ we have $(H x)(t)=(H y)(t)$ whenever $x$ and $y$ satisfy $x(\tau)=y(\tau)$ for $\tau \leq t$. Our main result is the following, in which $B L_{1}(\mathbb{R})$ stands for the set of Lebesgue-integrable elements of $L_{\infty}(\mathbb{R})$.

Theorem 1: There exists a causal $H \in \mathcal{H}$ with the following properties.

(i) $(H x)(t)=0, \quad t \in \mathbb{R}$ for each $x \in B L_{1}(\mathbb{R})$.

(ii) $(H x)(t)=\lim _{\alpha \rightarrow-\infty} x(\alpha), \quad t \in \mathbb{R}$ for each $x \in L_{\infty}(\mathbb{R})$ for which the limit on the right side exists.

This immediately yields the following, because $L_{\infty}(\mathbb{R})$ clearly contains elements $x$ for which $\lim _{\alpha \rightarrow-\infty} x(\alpha)$ exists and is nonzero.

Corollary: There exists a causal $H \in \mathcal{H}$ for which

(a) $H$ has an impulse response, and this impulse response is the zero function.

(b) There are elements $x$ of $L_{\infty}(\mathbb{R})$ such that $H x$ is not the zero function.

\footnotetext{
${ }^{4}$ More specifically, with $q(0)=\infty$ allowed, the integral is zero as a Lebesgue integral or an improper Riemann integral. In the remainder of the paper, all integrals are meant to be interpreted as Lebesgue integrals.
}

\section{Proof of Theorem 1:}

We will use the following result which is proved (but not formally stated) in [1]. For the reader's convenience, a proof is given in the Appendix.

Lemma 1: There exists a continuous linear shiftinvariant causal ${ }^{5}$ map $E$ from $\mathcal{C}$ into itself such that

$$
(E x)(t)=\lim _{\alpha \rightarrow-\infty} x(\alpha), \quad t \in \mathbb{R}
$$

for each $x \in \mathcal{C}$ for which the limit on the right side exists.

Let $F$ denote the map from $L_{\infty}(\mathbb{R})$ into $L_{\infty}(\mathbb{R})$ defined by

$$
(F x)(t)=\int_{\mathbb{R}} f(t-\tau) x(\tau) d \tau, \quad t \in \mathbb{R}
$$

in which $f \in B L_{1}(\mathbb{R})$. Since

$$
\begin{gathered}
\left|(F x)\left(t_{1}\right)-(F x)\left(t_{2}\right)\right| \leq\|x\| \int_{\mathbb{R}}\left|f\left(t_{1}-\tau\right)-f\left(t_{2}-\tau\right)\right| d \tau \\
=\|x\| \int_{\mathbb{R}}\left|f(\tau)-f\left(t_{2}-t_{1}+\tau\right)\right| d \tau
\end{gathered}
$$

in which the last integral approaches zero as $\left|t_{2}-t_{1}\right| \rightarrow$ 0 , because $f$ is integrable, we see that $F$ in fact maps into $\mathcal{C}$.

Lemma 2: If $x \in L_{\infty}(\mathbb{R})$ satisfies $\lim _{\alpha \rightarrow-\infty} x(\alpha)=\zeta$ for some $\zeta$, then

$$
\lim _{t \rightarrow-\infty}(F x)(t)=\zeta \int_{\mathbb{R}} f(\tau) d \tau .
$$

\section{Proof of Lemma 2:}

Suppose that $x$ is as indicated. We have

$$
\begin{gathered}
(F x)(t)=\int_{\mathbb{R}} f(t-\tau) x(\tau) d \tau \\
=\zeta \int_{\mathbb{R}} f(\tau) d \tau+\int_{\mathbb{R}} f(t-\tau)[x(\tau)-\zeta] d \tau
\end{gathered}
$$

for each $t$. Consider the last integral, and let any $\epsilon>0$ be given. Using the hypothesis that $f$ is integrable, choose a negative $c_{1}$ so that

$$
\sup _{\alpha<c_{1}}|x(\alpha)-\zeta| \int_{\mathbb{R}}|f(\tau)| d \tau<\epsilon / 2
$$

and then, using the fact that

$$
\int_{\tau \geq c_{1}}|f(t-\tau)| d \tau=\int_{-\infty}^{\left(t-c_{1}\right)}|f(\tau)| d \tau
$$

select a $c_{2}<0$ for which

$$
\sup _{\alpha \in \mathbb{R}}|x(\alpha)-\zeta| \int_{\tau \geq c_{1}}|f(t-\tau)| d \tau<\epsilon / 2
$$

\footnotetext{
$\mathcal{H}$.
} 
for $t<c_{2}$. Observe that for $t<c_{2}$,

$$
\begin{gathered}
\left|\int_{\mathbb{R}} f(t-\tau)[x(\tau)-\zeta] d \tau\right| \leq \int_{\tau<c_{1}}|f(t-\tau)[x(\tau)-\zeta]| d \tau \\
\quad+\int_{\tau \geq c_{1}}|f(t-\tau)[x(\tau)-\zeta]| d \tau<\epsilon / 2+\epsilon / 2=\epsilon,
\end{gathered}
$$

which proves the lemma.

\section{Lemma 3:}

$$
\lim _{|t| \rightarrow \infty} \int_{\mathbb{R}} f(t-\tau) x(\tau) d \tau=0
$$

for each $x \in B L_{1}(\mathbb{R})$.

\section{Proof of Lemma 3:}

Both $f$ and $x$ belong to $L_{2}(\mathbb{R})$. Using a version of the Parseval identity,

$$
\begin{gathered}
2 \pi \int_{\mathbb{R}} f(t-\tau) x(\tau) d \tau \\
\left.=\int_{\mathbb{R}} \exp \{j \omega t)\right\} \hat{f}(\omega) \hat{x}(\omega) d \omega, \quad t \in \mathbb{R}
\end{gathered}
$$

in which $j=\sqrt{-1}$, and $\hat{f}$ and $\hat{x}$ denote the Fourier transforms of $f$ and $x$, respectively. These Fourier transforms belong to $L_{2}(\mathbb{R})$. By the Schwarz inequality, $z$ given by $z(\omega)=\hat{f}(\omega) \hat{x}(\omega)$ for all $\omega$ is integrable. Therefore, by the Riemann-Lebesgue lemma, the right side of (3) approaches zero as $|t| \rightarrow \infty$, proving the lemma.

Continuing with the proof of the theorem, assume now that $f(t)=0$ for $t<0$, and that

$$
\int_{0}^{\infty} f(t) d t=1
$$

With $E$ as described in Lemma 1, set $H=E F$. We see that $H$ is a causal linear shift-invariant continuous map of $L_{\infty}(\mathbb{R})$ into $\mathcal{C}$. By Lemmas 1 and 3, part (i) of the theorem holds. Using Lemmas 1 and 2, we see that part (ii) also holds.

\subsection{Comments}

The related result in [1] described in the Introduction concerns the properties of linear-system maps that take $\mathcal{C}$ into itself. Our theorem provides also a slight improvement of that result: In [1], the system impulse response is defined in terms of a sequence of continuous unit-integral inputs that vanish at $\infty$ and $-\infty$. By considering the restriction to $\mathcal{C}$ of the map $H$ of our theorem, we see that the conclusion of the result in [1] holds even without the condition that the unit-integral inputs vanish at $\infty$ and $-\infty$. (It is well known that the integrability of a function over $\mathbb{R}$ does not imply that it vanishes at $\infty$ and $-\infty$.)

Another implication of the material discussed concerns the frequency response of linear systems: It is a wide-spread belief that the input-output properties of linear shift-invariant systems are completely described by their frequency-domain response functions, ${ }^{6}$ i.e.,

\footnotetext{
${ }^{6}$ See, for example, [11, pp. 171-175].
}

by their response to the function $\exp \{j \omega \cdot\}$ for general $\omega \in \mathbb{R}$ where $j=\sqrt{-1}$, assuming of course that this response exists. In the setting of maps from $\mathcal{C}$ into itself, or from $L_{\infty}(\mathbb{R})$ to $\mathcal{C}$, the response clearly exists, and is it known to be given by

$$
[H \exp \{j \omega \cdot\}](t)=\exp \{j \omega t\} K(\omega), \quad t \in \mathbb{R}^{7}
$$

for each $\omega$, in which $K(\omega)=[H \exp \{j \omega \cdot\}](0)$. Thus, according to the the wide-spread belief, the response of $H$ is completely described by the function $K$. But this too was recently found $[12]^{8}$ to be contrary to the facts: there is a continuous $E: \mathcal{C} \rightarrow \mathcal{C}$ of the kind described for which $K$ is the the zero function, but $E$ takes any nonzero $x \in \mathcal{C}$ such that $x(t) \rightarrow 0$ as $|t| \rightarrow \infty$ into a nonzero element of $\mathcal{C}$.

By considering maps $H$ of the form $E F$, where $F: L_{\infty}(\mathbb{R}) \rightarrow \mathcal{C}$ is given by $(2)$ and $f \in B L_{1}(\mathbb{R})$ is such that its Fourier transform never vanishes, and using Lemma 3, we arrive at the following concerning continuous maps whose domain is all of $L_{\infty}(\mathbb{R})$.

Theorem 2: There exists an $H \in \mathcal{H}$ with the following properties.

(i) $H \exp \{j \omega \cdot\}$ is the zero function for each $\omega \in \mathbb{R}$.

(ii) $H x$ is a nonzero element of $\mathcal{C}$ for every element $x$ of $B L_{1}(\mathbb{R})$ with positive $L_{1}(\mathbb{R})$ norm.

\section{Appendix: Proof of Lemma 1}

We first introduce some definitions and prove a proposition.

Let $L$ denote a linear manifold in $\mathcal{C}$ that is closed under translation in the sense that $T_{\tau} L=L$ for each $\tau \in \mathbb{R}$, where $T_{\tau}$ is the usual shift map defined on $L$ for each $\tau$ by $\left(T_{\tau} x\right)(t)=x(t-\tau), t \in \mathbb{R}$. We do not rule out the possibility that $L=\mathcal{C}$.

Let $Q$ from $\mathcal{C}$ into itself be defined by $(Q x)(t)=$ $x(t), t \leq 0$ and $(Q x)(t)=x(0), t>0$. Assume that $L$ is closed under $Q$.

Let $A$ be a linear map of $L$ into $\mathcal{C}$. Such an $A$ is shift invariant (or, equivalently, time invariant) if

$$
(A x)(t-\tau)=\left(A T_{\tau} x\right)(t), \quad t \in \mathbb{R}
$$

for each $\tau \in \mathbb{R}$ and $x \in L$. It is bounded if $\|A\|_{L}:=$ $\sup \{\|A x\|: x \in L,\|x\| \leq 1\}<\infty$. Finally, by $A$ is causal is meant that $A$ is causal in the usual sense. ${ }^{9}$ Our proposition is the following.

Proposition: Let $A$ be shift invariant, causal, and bounded. Then there exists a bounded (and thus continuous) linear shift-invariant causal map $E$ from $\mathcal{C}$ into itself that extends $A$ in the sense that $E x=A x, x \in L$.

\section{Proof of the Proposition:}

We will use the following observation.

\footnotetext{
${ }^{7}$ By the linearity and shift-invariance of $H$, and for any $t \in \mathbb{R}$, we have $\exp \{-j \omega t\}[H \exp \{j \omega \cdot\}](t)=$ $[H \exp \{j \omega(\cdot-t)\}](t)=[H \exp \{j \omega \cdot\}](0)$, which gives $(4)$.

${ }^{8}$ An erratum appears in vol. 21, p. 450, 2002.

${ }^{9}$ Of course, this means that for each $t$, we have $(A x)(t)=$ $(A y)(t)$ whenever $x$ and $y$ satisfy $x(\tau)=y(\tau)$ for $\tau \leq t$.
} 
Subproposition: Let $A$ be shift invariant. Then $A$ is causal if and only if $A(\cdot)(0)=A(Q \cdot)(0)$ on $L$.

\section{Proof of the Subproposition:}

Suppose that $A(\cdot)(0)=A(Q \cdot)(0)$ on $L$. Let $t \in \mathbb{R}$ and let $x, y \in L$ be such that $x(\tau)=y(\tau)$ for $\tau \leq t$. Using the shift-invariance of $A$, we have $(A x)(t)=$ $\left(A T_{-t} x\right)(0)=\left(A Q T_{-t} x\right)(0)$, and similarly $(A y)(t)=$ $\left(A Q T_{-t} y\right)(0)$. Since $Q T_{-t} x=Q T_{-t} y, A$ is causal.

Now suppose that $A$ is causal, and let $x \in L$ be arbitrary. Then $(A x)(0)=(A Q x)(0)$ because $x(t)=$ $(Q x)(t), t \leq 0$, which proves the subproposition.

The rest of the proof of the proposition is similar to a proof in [4]: We have $(A x)(t)=\left(A T_{-t} x\right)(0)$ for all $t$ and all $x \in L$. The map $(A \cdot)(0)$ is a bounded linear functional on $L$, because

$$
\begin{gathered}
|(A y)(0)|=\left|\left(A T_{-t} T_{t} y\right)(0)\right|=\left|\left(A T_{t} y\right)(t)\right| \leq \\
\sup _{\beta}\left|\left(A T_{t} y\right)(\beta)\right| \leq\|A\|_{L} \cdot\left\|T_{t} y\right\|=\|A\|_{L} \cdot\|y\|
\end{gathered}
$$

for $y \in L$. By the Hahn-Banach theorem [9, pp. 178 and 181] there is a bounded linear functional $\mathcal{F}$ that extends $(A \cdot)(0)$, which equals $A(Q \cdot)(0)$, to all of $\mathcal{C}$. Define $E$ on $\mathcal{C}$ by $(E x)(t)=\mathcal{F} Q T_{-t} x$. We see that $E$ is linear. It maps into the set of complex-valued bounded functions on $\mathbb{R}$ because

$$
\left|\mathcal{F} Q T_{-t} x\right| \leq\|\mathcal{F}\| \cdot\left\|Q T_{-t} x\right\| \leq\|\mathcal{F}\| \cdot\|x\|
$$

for $t \in \mathbb{R}$. Using the fact that the map $T_{(\cdot)} x$ from $\mathbb{R}$ to $\mathcal{C}$ is uniformly continuous for each $x \in \mathcal{C}$, it is easy to check that $E$ is a shift-invariant bounded map into $\mathcal{C}$. Also, $E(\cdot)(0)=\mathcal{F} Q$ and $E(Q \cdot)(0)=\mathcal{F} Q Q=$ $\mathcal{F} Q$, showing (by the subproposition) that $E$ is causal. Since $E$ extends $A$ to $\mathcal{C}$, this completes the proof of our proposition.

Continuing with the proof of the lemma, let $L$ be the family of all functions $x$ in $\mathcal{C}$ such that such that $\lim _{t \rightarrow-\infty} x(t)$ exists. Take $A$ to be the linear map of $L$ into $\mathcal{C}$ defined by

$$
(A x)(t)=\lim _{\alpha \rightarrow-\infty} x(\alpha), \quad t \in \mathbb{R} .
$$

We see that $L$ is a linear manifold that is closed under translation and under $Q$, and that $A$ is linear, shift invariant, and causal. In addition, $A$ is bounded because

$$
|(A x)(t)|=\left|\lim _{\alpha \rightarrow-\infty} x(\alpha)\right| \leq\|x\|, \quad t \in \mathbb{R}
$$

for $x \in L$. By our proposition, there is a continuous linear shift-invariant causal map $E$ from $\mathcal{C}$ into itself that extends $A$. Since (5) holds, this completes the proof of Lemma 1.

\section{References:}

[1] I. W. Sandberg, "Causality and the Impulse Response Scandal," IEEE Transactions on Circuits and Systems I, vol. 50, no. 6., pp. 810-811, 2003.

[2] C. Corduneanu, Almost Periodic Functions, New York: Interscience Publishers, 1961.

[3] I. W. Sandberg, "Notes on Representation Theorems for Linear Discrete-Space Systems," Proceedings of the International Symposium on Circuits and Systems, Orlando, Florida, May 30-June 2, 1999 (four pages on $\mathrm{CD}$ ).

[4] I. W. Sandberg, "Multidimensional Nonlinear Myopic Maps, Volterra Series, and Uniform NeuralNetwork Approximations," pp. 99-128 in D. Docampo, A. Figueiras-Vidal, and F. Perez-Gonzalez (eds.), Intelligent Methods in Signal Processing and Communications, Boston: Birkhauser, 1997.

[5] W. J. Borodziewicz, K. J. Jaszczak, and M. A. Kowalski, "A Note on Mathematical Formulation of Discrete-Time Linear Systems," Signal Processing, vol. 5, pp. 369-375, 1983.

[6] P. Hughett, "Representation Theorems for Semilocal and Bounded Linear Shift-Invariant Operators on Sequences," Signal Processing, vol. 67, pp. 199-209, 1998.

[7] I. W. Sandberg, "Multidimensional Linear Systems: the Extra Term," International Journal of Circuit Theory and Applications, vol. 27, pp. 415-420, 1999.

[8] R. E. Edwards, Functional Analysis, New York: Dover, 1995.

[9] G. Bachman and L. Narici, Functional Analysis, New York: Academic Press, 1966.

[10] M. J. Lighthill, Introduction to Fourier Analysis and Generalized Functions, Cambridge: Cambridge University Press, 1960.

[11] W. B. Davenport, Jr. and W. L. Root, Random Signals and Noise, New York: McGraw-Hill, 1958.

[12] I. W. Sandberg, "Continuous-Time Linear Systems: Folklore and Fact," Circuits, Systems and Signal Processing, vol. 21, no. 3, pp. 337-343, 2002. 